\title{
Effect of Motor Control Exercises on Pain and Mobility in Professional Drivers Having Occupational Low Back Pain
}

\author{
Vyom Gyanpuri, MPT ${ }^{1}$;Siddhartha Sen, MPT²; Neetu R Dhiman, MPT ${ }^{3}$; \\ Ajita, MPT ${ }^{4}$ \\ ${ }^{1}$ Kings Physiotherapy Center, Varanasi (Uttar Pradesh), India; \\ ${ }^{2}$ Assistant Professor, Department of Physiotherapy, Sardar Bhagwan Singh Post Graduate Institute of \\ Biomedical Sciences \& Research, Dehradun (Uttarakhand), India; \\ ${ }^{3}$ Department of Anatomy, Institute of Medical Sciences, Banaras Hindu University, \\ Varanasi (Uttar Pradesh), India. \\ ${ }^{4}$ Assistant Professor, Patiala University, Patiala (Punjab), India.
}

\begin{abstract}
:
Background and objective: The motor control exercises have been found to be effective in activating the deep trunk muscles. The aim of this study was to determine the effect of motor control exercise (stabilization exercise) on pain and mobility in professional drivers having Occupational low back pain.

Design: Experimental study design

Methods: Thirty male subjects having low back pain with the history of driving and age between 25 and 40 years were recruited from auto and taxi stands in Dehradun. Subjects were divided into two groups: one is Group A and another is Group B each having 15 subjects. Subjects in group A were given the motor control exercises and back extension exercises while subjects in group $B$ were given only the back extension exercises. This was a four week exercise program. Pain and Performance were assessed Pre-intervention, Midintervention and Post-intervention using Visual Analogue Scale (VAS) and Back Performance Scale (BPS) respectively. Within-group and between-group comparison of all three readings was done.

Results: The results showed insignificant improvement in pain and performance of both the groups from the 0 day to $4^{\text {th }}$ week of intervention. The difference between the improvements shown by both groups was also found to be insignificant.

Conclusion: From the above results, it was concluded that the motor control exercises are equally effective to other back extension exercises in reducing pain and increasing mobility in professional drivers having occupational LBP.
\end{abstract}

Keywords: Low back pain, motor control exercise

\section{INTRODUCTION}

Low back pain (LBP) is recognized as the leading cause of occupational injury in developed countries. There is strong evidence, however, that approximately $10 \%$ of the cases cause more than $80 \%$ of the cost for low back pain because of their chronicity. At this writing, most research efforts have been directed at investigating risk factors for the incidence or reporting of acute back pain. ${ }^{1}$ Low back pain is among the most frequent health problems in the general population, affecting 58-84\% of all adults at some point in their life.Also, disabling LBP is responsible for substantial costs to both the individual patient and society. ${ }^{2}$ Low back pain is one of the main causes of disability, and, despite its high prevalence, the source of pain is not established in the majority of cases and the term "nonspecific low back pain" is used. ${ }^{3}$ One factor that has been proposed as important in the genesis and persistence of nonspecific LBP is stability and control of the spine. ${ }^{4}$ Sitting has been associated with risk of developing LBP. ${ }^{5}$

Driving is a part of our life, such that it is difficult to advise 'giving up' driving or, due to cost, to advise 'changing vehicle' in order to investigate whether driving a particular vehicle is causing musculoskeletal troubles. There are many reasons why a prevalence of back pain could be expected, for example prolonged sitting, fixed posture, vibration, loss of lumbar lordosis, asymmetric forces acting on the spine and perhaps periodic lifting, any which individually could lead to musculoskeletal troubles. ${ }^{6}$ Jiu-Chiuan Chen studied on Occupational factors associated with low back pain in urban taxi drivers. He aimed to examine low back pain in taxi drivers and its association with prolonged driving and other occupational factors. He found of 1242 drivers, $51 \%$ reported LBP in the past 12 months, significantly higher than other professional drivers $(33 \%)$ in Taiwan. They concluded that the high Low back pain frequency in taxi drivers was associated with long driving time, frequent bending/twisting activities while driving, self perceived job stress, job dissatisfaction and registration type. 
In keeping with Panjabi's theory, changes in recruitment of specific deep spinal muscles thought to be responsible for the control of spinal stability have been reported in people with spinal pain. The onset of the activity of the abdominal muscle, transverse abdominis, is delayed in people with LBP. ${ }^{8}$ A significant body of research shows that, the atrophy seen in the multifidus muscle in people with low back dysfunction is representative of a form of impaired motor control, not simple disuse weakness. As such, traditional strengthening exercises will often fail to correct this fault. ${ }^{9}$

Motor control exercises called as specific stabilization exercise for low back pain is a widely used form of exercise and there is an extensive body of literature that provides a rationale for the mechanism of action. The primary aim of motor control exercise is to re-establish normal activity of the deep spinal muscle, reducing activity of more superficial muscle that tend to stiffen the spine and have increased activity in low back pain and then maintain normal control during progressively more demanding physical and functional tasks. The key feature of the motor control exercise approach is the training of the deep trunk muscles in isolation before progressing to demanding tasks that train coordination of the deep and the superficial trunk muscles. ${ }^{10}$ Stabilization training follows the basic principles of motor learning first by developing awareness of muscle contraction and spinal position, then developing control in simple patterns and exercises and progressing to complex, and finally demonstrating automatic maintenance of spinal stability and in a progression of simple functional activities to complex and unplanned situation. ${ }^{11}$ This supports an integrated model of joint function and leads to optimal length-tension ratios and optimal force coupling of the muscles. Additionally, this model sets the stage for optimal postural alignment, normal movement patterns, and a minimal potential for joint dysfunction. ${ }^{12}$ Although some researcher have studied the effect of motor control exercise in patients with LBP, but no consensus literature has been found to determine objectively the effectiveness of motor control exercises in back pain due to occupational postural stress or dysfunction. Purpose of the study was to determine the effect of motor control exercise (stabilization exercise) on pain and mobility in professional drivers having Occupational low back pain.

\section{MATERIAL AND METHOD}

This study was performed on 30 male subjects (professional drivers) selected with convenient sampling after signing the informed consent form. TABLE 1 represents the demographic data for all subjects. Subjects were recruited from the Auto and taxi stands in Dehradun. . In order to participate in the study, subjects needed to be professional Male drivers (driving atleast 8 hours per day) between the age of 25 to 40 years and having low back pain since six months. ${ }^{6,7}$ We excluded volunteers from the study if they had any previous spinal surgery done, previous spinal trauma, subjects having radiating pain and hamstring tightness. We also excluded those subjects who had any spinal deformity.

Table1. Demographic Data

\begin{tabular}{|l|c|c|c|}
\hline & \multicolumn{3}{|c|}{ Mean \pm SD } \\
\cline { 2 - 4 } & Age & Weight & Height \\
\hline Group A & $34.4 \pm 4.50$ & $68.73 \pm 9.035$ & $167.33 \pm 7.87$ \\
\hline Group B & $34.4 \pm 3.92$ & $66.86 \pm 5.39$ & $164.5 \pm 6.35$ \\
\hline
\end{tabular}

\subsection{Instrumentation and Outcome measure}

\subsubsection{Visual analog scale (VAS)}

The VAS was used to assess each patient's pain perception (Fig. 1). It is a responsive pain scale that yields reliable and valid data. VAS rated on an intensity scale from 0 to $10 \mathrm{~cm}$, with higher scores representing higher levels of pain. VAS is usually a horizontal line, $10 \mathrm{~cm}$ in length. The patient marks on the line the point that they feel represents their perception pain. The VAS score is determined by measuring centimeters from the left hand end of the line to the point that the patient marks. ${ }^{13}$

Figure1. Visual analogue scale

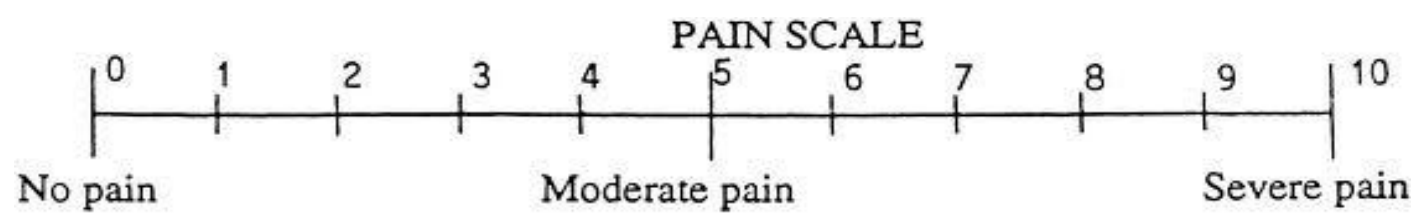




\subsubsection{Back performance scale (BPS)}

Activity limitation was assessed using BPS. Discriminative validity and responsiveness to important change are known for each of the tests. BPS was more responsive than any other tests for mobility related activities. The BPS is a reliable and valid outcome measure of activity limitation. Intertester reliability of the Back Performance Scale is very high with Intraclass correlation coefficient of 0.996. Test-retest reliability is also high with Intraclass correlation coefficient of $0.91 .{ }^{14} \mathrm{BPS}$ requirement: Chair, piece of paper, measuring tape, sand bag and table. It consists of 5 tests (Sock Test, Pick-up Test, Roll-up Test, Finger tip-to-Floor Test, and Lift Test), all requires sagittal-plane mobility. In all tests, performance is assessed using a 4-point ordinal scale. $^{15,16}$

\subsubsection{Sock Test}

From a sitting position: grab the toes with fingertips, the leg flexed in the sagittal plane, one leg tested at one time, scoring the least reach.

\subsubsection{Pick-up Test}

From a standing position: pick up a piece of paper from the floor, 2 or 3 times in varied ways.

\subsubsection{Roll-up Test}

From a supine position: roll up slowly into a long- sitting position, arms relaxed.

\subsubsection{Fingertip-to-Floor Test}

From a standing position: feet $10 \mathrm{~cm}$ apart and knees straight, reach toward the floor with fingertips.

\subsubsection{Lift Test from a standing position}

Repeat lifting a box containing a sandbag of $5 \mathrm{~kg}$, for $1 \mathrm{~min}$, from the floor to the table.

\subsection{Protocol}

Thirty subjects were selected from the auto and taxi stands based on inclusion \& exclusion criteria. Subjects were divided into two groups: one is Group A and another is Group B, each having 15 subjects. Subjects in group A were given the motor control exercises plus the back extension exercises while subjects in group B were given only the back extension exercises only. Pain and Performance were assessed three times using VAS and BPS respectively: Pre-intervention, Mid-intervention, and Post-intervention. Within-group and between-group comparison of all three readings were done.

\subsection{Procedure}

Whole procedure was explained to each subject and then the informed consent was obtained by them. The pain and mobility score of each subject were assessed using VAS and BPS scale respectively, pre intervention (0 day), mid intervention ( $15^{\text {th }}$ day) and post intervention $\left(30^{\text {th }}\right.$ day) of both groups (Group A and Group B).

All subjects were given a 20 minute training session on the very first day of intervention program. This included three training activities which helped in increasing abdominal muscle awareness before preintervention. Abdominal breathing in the supine position was used to provide an increase in general awareness of the abdominal muscles and breathe control. Quadruped abdominal hollowing in which gravity was presumed to provide resistance to Transverse Abdominis contraction while the subject attempted to hollow the abdomen. ${ }^{17,} 18$ The third activity was abdominal hollowing in the hook-lying position. This was done using sphygmomanometer (For visual feedback). The sphygmomanometer cuff was placed beneath the back at the level of umbilicus. The cuff was inflated upto $40 \mathrm{mmh}$ and the aneroid meter was handed to the subject so that it is visible to the subject. Then, the subject was instructed to hollow the abdomen and increase or maintain the pressure at $40 \mathrm{mmHg}( \pm 10 \mathrm{mmHg})$ for 10 seconds. ${ }^{10,19}$

\subsubsection{Group A}

Below are the exercises performed by the subjects in group A and the motor control exercises were changed weekly while back extension exercises were kept the same.

\subsubsection{First Week}

2.3.1.1.1Drawing in maneuver while maintaining normal respiration

Subjects were in hook lying position. Subjects were taught to draw in the abdominal wall without moving the spine or pelvis and hold the same position while maintaining the normal respiration process (Fig. 2). 


\subsection{Prone with alternate leg raise}

Subjects were in prone lying position. Subjects were instructed to raise their legs alternatively. ${ }^{20}$

\subsection{Prone with chest raise}

Subjects were in prone lying position. Subjects were taught to elevate their upper body on their elbows. ${ }^{20}$

\subsubsection{Second Week}

2.3.1.2.1 Four point kneeling and trying to hollow the lower abdomen

Subjects were in quadruped position. Subjects were taught to draw in the abdominal wall without moving the spine or pelvis and hold the same position while maintaining the normal respiration process (Fig. 3).

\subsection{Prone with alternate leg raise}

\subsection{Prone with chest raise}

\subsubsection{Third Week}

\subsection{Heel slides with Drawing in maneuver in crook lying position}

Subjects were asked to lie in hook lying position. Subjects were taught to draw in the abdominal wall without moving the spine or pelvis and hold the same position while maintaining the normal respiration process and told to slide the heel alternatively (Fig. 4).

\subsection{Prone with alternate leg raise}

2.3.1.3.3 Prone with chest raise

\subsubsection{Fourth Week}

\subsection{Single hip abduction while maintaining abdominal hollowing}

Subjects were in hook lying position. Subjects were taught to draw in the abdominal wall without moving the spine or pelvis and hold the same position while maintaining the normal respiration process and abducting the hip alternatively (Fig. 5).

2.3.1.4.2 Prone with alternate leg raise

2.3.1.4.3 Prone with chest raise

Figure2. Drawing in maneuver while maintaining normal respiration

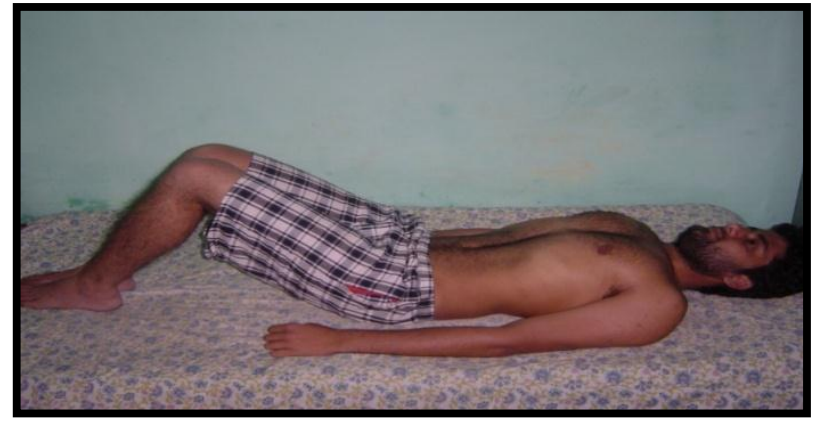

Figure3. Four point kneeling and trying to hollow the lower abdomen

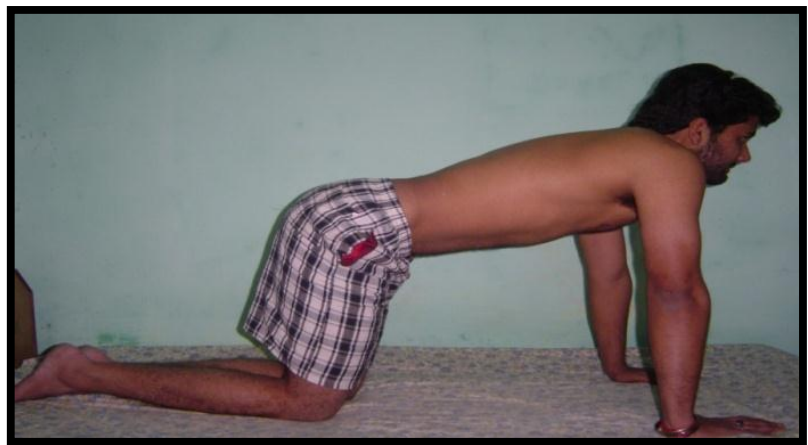


Figure4. Heel slides with Drawing in maneuver in crook lying position

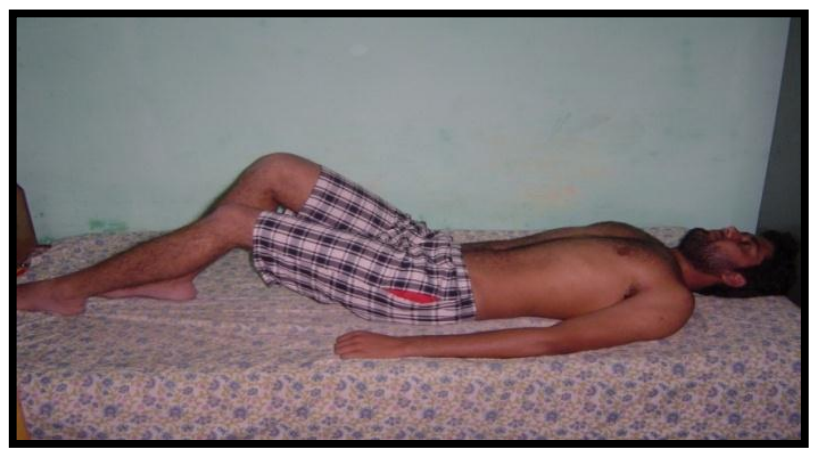

Figure5. Single hip abduction while maintaining abdominal hollowing

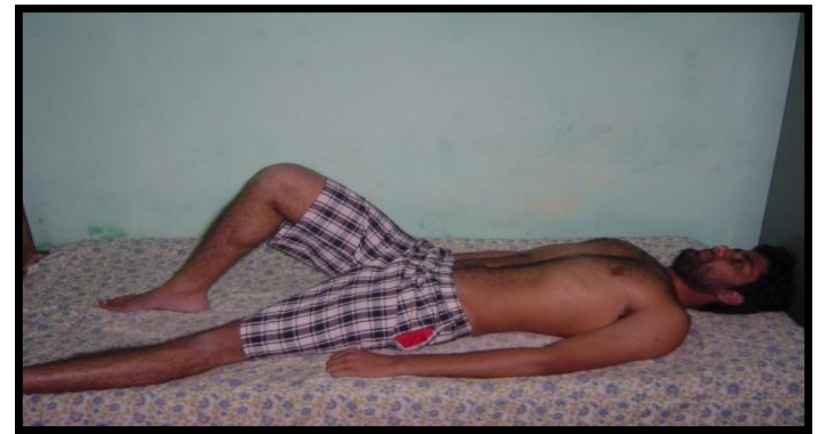

\subsubsection{Group B}

Subjects in group B were given only the back extension exercises explained earlier.

2.3.2.1 Prone with alternate leg raise

2.3.2.2 Prone with chest raise

Figure6. Study procedure

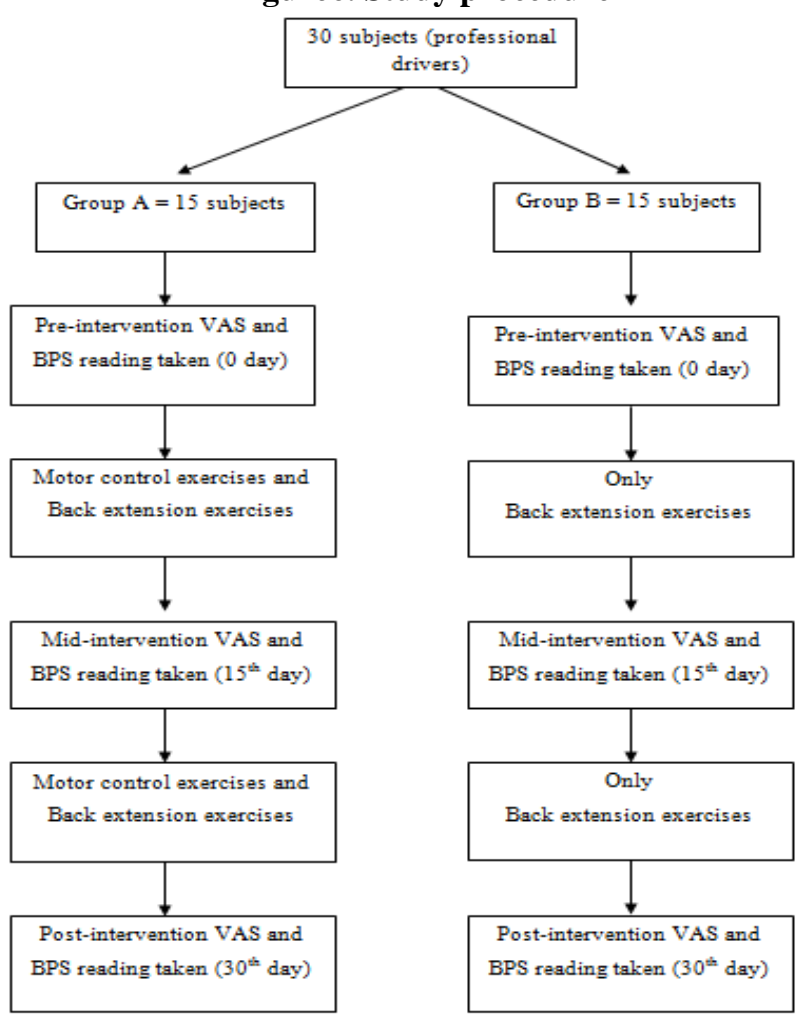


The intervention was of four weeks in which exercises were followed daily (once a day). Subjects were monitored carefully while performing the exercises for the assurance of correct performance. Every exercise was performed in a set of 10 repetitions with 10 seconds hold and 10 seconds rest period in between. Three readings of BPS and VAS were taken i.e. Pre-intervention, Mid-intervention and Post-intervention (Fig. 6).

\section{DATA ANALYSIS}

The data was analyzed by using Statistical Package of Social Science-SPSS software (version 11.5). The arithmetic mean and standard deviation of the age, weight and height were calculated. One way ANOVA test was applied to compare the means of VAS and BPS scoring of pre-intervention, mid-intervention and postintervention within groups. Post-hoc analysis of multiple comparison tests was applied for pair wise mean comparison. Independent t-test was used for between group comparison of both VAS and BPS scoring of all three intervention readings. The significant level was set at $5 \%(\mathrm{p}=0.05)$

\section{RESULTS}

Within group analysis revealed that there is some improvement in pain and performance from 0 week to 4 th week of intervention in both groups but the difference is not statistically significant.(TABLE 2 ).

Between groups analysis showed that mean pre, mid and post intervention reading of group B (VAS) is less than mean pre, mid and post-intervention reading of group A but again, the difference is not statistically significant. There is not much increase in the means of all three intervention readings of BPS from Group A to Group B. (TABLE 3).

Table2. ANOVA of Pre, Mid and Post Intervention within Group A and Group B

\begin{tabular}{|c|c|c|c|c|c|}
\hline & $\begin{array}{c}\text { Pre-intervention } \\
\text { (0 day) } \\
(\text { mean } \pm \text { SD })\end{array}$ & $\begin{array}{c}\text { Mid-intervention } \\
\left(15^{\text {th }} \text { day }\right) \\
(\text { mean } \pm \text { SD })\end{array}$ & $\begin{array}{c}\text { Post-intervention } \\
\left(\mathbf{3 0}^{\text {th }} \text { day }\right) \\
(\text { mean } \pm \text { SD })\end{array}$ & $\mathbf{F}$ & Sig. \\
\hline Group A (VAS) & $4.33 \pm 2.05$ & $3.66 \pm 2.16$ & $3.13 \pm 1.92$ & 1.291 & 0.286 \\
\hline Group B (VAS) & $4.4 \pm 1.35$ & $3.93 \pm 1.48$ & $3.73 \pm 1.22$ & 0.952 & 0.712 \\
\hline Group A (BPS) & $6.2 \pm 3.78$ & $5.66 \pm 3.37$ & $5.13 \pm 3.41$ & 0.342 & 0.394 \\
\hline Group B (BPS) & $6.06 \pm 3.43$ & $5.8 \pm 3.27$ & $5.73 \pm 3.19$ & 0.043 & 0.958 \\
\hline
\end{tabular}

Table3. T-Test of Pre, Mid and Post Intervention between Group A and Group B

\begin{tabular}{|c|c|c|c|c|}
\hline & & $\begin{array}{l}\text { Mean difference } \\
\pm \text { Standard error }\end{array}$ & $\mathbf{t}$ & $p$ value \\
\hline \multirow{3}{*}{$\begin{array}{c}\text { Group-A } \\
\text { Versus } \\
\text { Group-B }\end{array}$} & VAS-0 DAY & $0.22 \pm 0.64$ & 0.343 & 0.734 \\
\hline & VAS- $15^{\text {th }}$ DAY & $0.22 \pm 0.69$ & 0.312 & 0.750 \\
\hline & VAS- $30^{\text {th }}$ Day & $0.86 \pm 0.58$ & 1.481 & 0.155 \\
\hline \multirow{3}{*}{$\begin{array}{l}\text { Group-A } \\
\text { Versus } \\
\text { Group-B }\end{array}$} & BPS-0 DAY & $0.61 \pm 1.34$ & 0.456 & 0.652 \\
\hline & BPS- $15^{\text {th }}$ DAY & $0.027 \pm 1.23$ & 0.022 & 0.982 \\
\hline & BPS- 30 $^{\text {th }}$ Day & $0.44 \pm 1.23$ & 0.360 & 0.722 \\
\hline
\end{tabular}

AS- Visual Analogue Scale

BPS- Back Performance Scale

\section{DISCUSSION}

The within group comparison showed no significant changes in the improvement of pain and mobility in both groups. We also found no significant difference between Group A (motor control + back extension exercise) and Group B (back extension exercise). But, when the within group mean comparison of pre, mid and post intervention readings of VAS and BPS of both groups was done, then group A showed little improvement in both pain and mobility from 0 day to $4^{\text {th }}$ week of intervention which is not statistically significant. And this improvement is little more than the improvement in Group B when the between group analysis was done. Hence, we can say that the improvement in pain and mobility of Group A is equally significant to the improvement shown by the Group B. 
According to Asghar Akbar ${ }^{15}$, when he studied the effect of motor control exercises on lumbar local stabilizing muscle thickness in patients with chronic low back pain, he reported that motor control exercises decreased pain and increased TA (transverse abdominis) and LM (lumbar multifidi) muscles thickness and lumbar mobility in patients with chronic Low Back Pain (LBP) without any signs of spinal instability. He gave an intervention of eight weeks.

According to Peter B. O'Sullivan ${ }^{21}$, a specific exercise intervention (motor control exercise) is significantly effective in reducing pain intensity and functional disability levels in the treatment of patients with chronic low back pain. He found significant improvement in pain and function with these exercises, which were maintained at a 30 month follow up. The results of our study are not in support with the results of these studies and the reason for this might be the duration of the intervention period of our study which was very less as compared to the duration of the intervention period of these previous studies.

According to a study done by Luciana $\mathrm{G}$ Macedo ${ }^{4}$ et al, in which the effectiveness of motor control exercises for persistent low back pain was evaluated. They concluded that motor control exercise is superior to minimal intervention and confers benefit when added to another therapy for pain at all time points and for disability at long term follow up. They also reported that motor control exercise is not more effective than manual therapy or other forms of exercises. According to a systematic review of the efficacy of specific stabilization exercise (motor control exercise) for spinal and pelvic pain done by Paula $\mathrm{H}$ Ferreira ${ }^{8}$, the spinal stabilization exercises are effective in reducing pain and disability in chronic but not acute low back pain. The results of both of these studies are supported by the results of our study that motor control exercises are equally effective to other back exercises in reducing pain and improving mobility in patients with LBP.

Hides et al studied patients with acute, first-time episode of unilateral LBP, comparing stabilization exercises particularly targeting the multifidus muscle with usual medical management. No significant differences in disability or pain were found after 4 weeks, but the stabilization group experienced significantly fewer recurrences at 2- to 3-year follow-up. These studies indicated that a specific stabilization exercise approach may be effective for certain subgroups of patient. ${ }^{22}$ The review done by Paula $\mathrm{H}$ Ferreira ${ }^{8}$ mentioned before and a recent systematic review of exercise therapy for LBP showed the similar results that exercise therapy overall was not effective for patients with acute LBP but may be helpful for those with chronic LBP. The review also noted that the evidence of improvement could not be used to examine the effectiveness of specific type of exercise because of heterogeneity in study populations. ${ }^{22}$ Similarly, in our study also, we took the professional drivers having the back pain and there was no criteria for the chronicity or intensity of pain. So, our subjects were heterogeneous in relation to the intensity of pain and also our sample size was very small. These might be one of the reasons for very little improvement. The few studies that have examined specific stabilization exercise programs in more homogenous populations have shown more promising results. ${ }^{22}$ In our study, there was insignificant improvement in pain and performance in the drivers. Also there are some studies as we mentioned earlier, in which it has been shown that stabilization exercises have no significant effect on pain and disability in patients with LBP.

A future research can be done in which this intervention is extended for longer duration of time may be for example, for six months or one year and follow up can be done to see the long term effect of motor control exercises on pain and mobility in patients with low back pain. This study can be done on a large number of patients with low back pain due to other spinal disorders like disc prolapsed etc.

\section{CONCLUSION}

From the present study, it is concluded that the motor control exercises are equally effective to other back extension exercises in reducing pain and increasing mobility in professional drivers having occupational LBP. So, it can be said that instead of using specifically the motor control exercises, other back extension exercises can also be used for the treatment of patients with low back pain as both are equally effective according to our study.

\section{ACKNOWLEDGEMENT}

The authors thank all the candidates for their active participation in the study.

\section{Funding and Conflict of interest statement}

This research received no specific grant from any funding agency in the public, commercial, or notfor-profit sectors. The Authors declare that there is no conflict of interest. 


\section{REFERENCES}

[1] Marlene Fransen et al. Risk Factors Associated with the Transition from Acute to Chronic Occupational Back Pain. Spine 2002; 27(1): 92-98.

[2] A Van Nieuwenhuyse, P R Somville. The role of physical workload and pain related fear in the development of low back pain in young workers: evidence from the BelCoBack Study; results after one year of follow up. Occup Environ Med 2006; 63: 45-52.

[3] Hancock MJ, Maher CG. Systematic review of tests to identify the disc, SIJ or facet joint as the source of low back pain. Eur Spine J. 2007; 16:1539-1550.

[4] Luciana G Macedo and Christopher G Maher. Motor Control Exercise for Persistent, Nonspecific Low Back Pain: A Systematic Review. Physical Therapy 2009 Jan; 89(1): 9-25.

[5] Angela Maria Lis, M. Black. Association between sitting and occupational LBP Eur Spine J 2007; 16:283-298.

[6] J.M.Porter and D.E. Gyi. The Prevalence of Musculoskeletal troubles among car drivers. Occupational medicine 2002; 52(1): 4-12.

[7] Jiu-Chiuan Chen, Wen-Ruey Chang. Occupational factors associated with low back pain in urban taxi drivers. Occupational Medicine 2005; vol 55: 535-540.

[8] Paulo H Ferreira.Specificstabilisation exercise for spinal and pelvic pain: A systematic review. Australian Journal of Physiotherapy 2006; 52: 79-88.

[9] R S Jemmett. Rehabilitation of lumbar multifidus dysfunction in low back pain: strengthening versus a motor re-education model. Br J Sports Med 2003; 37: 91-94.

[10] Chris G Maher, Jane Latimer. The effect of motor control exercise versus placebo in patients with chronic low back pain. BMC Musculoskeletal Disorders 2005;6:54.

[11] Carolyn kisner, lynnallen Colby: Therapeutic Exercise foundation and Techniques. $5^{\text {th }}$ edition USA; F A Davis company2007: p. 657-60.

[12] Michael Fredericson,et al. Muscular Balance, Core Stability, and Injury Prevention for Middle- and Long-Distance Runners. Phys Med RehabilClin N Am 2005; 16: 669-689.

[13] D. Gould et al. Visual analog scale (VAS). Journal of Clinical Nursing 2001; 10: 697-706.

[14] MagnussenLiv, Strand Liv, Lygren. Reliability and Validity of the Back Performance Scale. Observing Activity Limitation in Patients with Back Pain. Spine. 2004 April 15; Vol 29 (8): 903-907.

[15] AsgharAkbari, SamaneKhorashadizadeh. The effect of motor control exercise versus general exercise on lumbar local stabilizing muscles thickness: Randomized controlled trial of patients with chronic low back pain. Journal of Back and Musculoskeletal Rehabilitation. 2008; 21: 105-112.

[16] LivInger Strand, Rolf Moe-Nilssen, Anne Elisabeth Ljunggren. Back Performance Scale for the Assessment of Mobility-Related Activities in People with Back Pain.Physical Therapy. 2002 Dec; 82(12): 1213-23.

[17] Marshall Hagins, Keri Adler et al. Effects of Practice on the Ability to Perform Lumbar Stabilization Exercises.Journal of Orthopaedic \& Sports Physical Therapy 1999; 29 (9): 546-55.

[18] C. Liebenson. Spinal stabilization training. Journal of body work and movement therapies 1997; 1(2): 87-90

[19] NitiRajpaletal.A Study on Efficacy of Pilates \& Pilates \&Mckenzie Exercises inPostural Low Back Pain: A Rehabilitative Protocol. Physiotherapy and Occupational Therapy Journal 2008; 1(1).

[20] KaulRohini, ThakralGaurav et al. Comparison of effects of specific stabilization exercises and Conventional back exercises in management of chronic Disc prolapse. Indian Journal of Physiotherapy and Occupational Therapy 2007 Sept; 1(3).

[21] O Sullivan, Peter B. et al. Evaluation of specific stabilization exercise in the treatment of chronic low back pain with radiologic diagnosis of spondylolysis or spondylolisthesis. Spine 1997 Dec 15; 22(24): 2959-67.

[22] Gregory E. Hicks, PhD, PT, Julie M. Fritz, PhD, PT et al. preliminary development of a clinical prediction rule for determining which patients with low back pain will respond to a stabilization exercise program. Arch Phys Med Rehabil 2005, Sept; 86: 175362. 